\title{
Emerging arboviruses (dengue, chikungunya, and Zika) in Southeastern Mexico: influence of socio-environmental determinants on knowledge and practices
}

\section{Arbovirosis emergentes (dengue, chikunguña y Zika) en el sudeste de México: la influencia de los determinantes socioambientales sobre el conocimiento y las prácticas}

\section{Arboviroses emergentes (dengue, chikungunya e Zika) no Sudeste do México: a influência dos determinantes socioambientais sobre o conhecimento e as práticas}

Roberta Causa 1,2

Héctor Ochoa-Díaz-López 3

Ariane Dor 4,5

Fernando Rodríguez-León 3

Roberto Solís-Hernández 3

Ana Laura Pacheco-Soriano 5

doi: 10.1590/0102-311X00110519

\begin{abstract}
The proliferation of arboviruses and their vectors is influenced by a complex interplay between vector, environment and human behaviors. The aim of this work is to analyze the influence of socio-environmental determinants on knowledge and practices regarding arboviruses transmission, among the residents of three communities on the southern border of Mexico. Between June 2017 and August 2018, a set of 149 households from three communities of Tapachula (Chiapas) and Villahermosa (Tabasco) were covered. This study consists of the application of a community prevention project. Different surveys and methodological approaches were used. Associations between so-

Correspondence

H. Ochoa-Díaz-López

El Colegio de la Frontera Sur.

Carretera Panamericana y Periférico Sur $s / n$, San Cristóbal de las Casas / Chiapas - 29290, México.

hochoa@ecosur.mx

1 Escuela Andaluza de Salud Pública, Granada, España. 2 Hospital Universitario de Puerto Real, Cádiz, España. 3 El Colegio de la Frontera Sur, San Cristóbal de las Casas, México.

4 Consejo Nacional de Ciencia y Tecnología, Tapachula, México. 5 El Colegio de la Frontera Sur, Tapachula, México.
\end{abstract} cio-environmental determinants and knowledge and practices for arboviruses transmission control were estimated by odds ratio. Logistic regression and qualitative techniques were used. Although around 75\% of households had an adequate knowledge about arboviruses' origin and transmission, only 30\% of them adopted adequate practices. Domestic risk practices were associated with serious deficiencies in water and sanitation services. Furthermore, a perception of greater risk and difficulty in complying with preventive measures were detected. An adequate knowledge does not necessarily lead to adequate prevention practices. Intermediate social determinants influence on the persistence of risk behaviors for arboviruses proliferation. Addressing such related aspects requires the achievement of an effective and sustainable vector management.

Arboviruses; Mosquito Control; Environment and Public Health; Sanitation 


\section{Introduction}

Dengue (DENV), Chikungunya (CHIKV) and Zika (ZIKV) are arthropod-borne viruses (arboviruses). In America, the Aedes aegypti mosquito is the primary vector of their transmission, a mosquito of tropical urban areas, widely adapted to domestic environments, and globally distributed 1,2 . Aedes albopictus is also present in America as a secondary vector of these diseases, transmitting the virus to different species of host through its blood-feeding behavior in rural or wild environments 3 .

In the Region of the Americas context, already endemic for DENV and with high Aedes infestation levels, the recent spread of CHIKV and ZIKV threatens and challenges the public health systems 4 .

In Mexico, autochthonous cases of CHIKV and ZIKV by Ae. aegypti transmission were detected for the first time in the southern border with Guatemala, near the city of Tapachula (Chiapas), in October 2014 and November 2015, respectively 5,6. In the state of Tabasco, first cases appeared between June 2015 and February 20167.

Since then, these diseases have been spreading northwards through the country. However, Mexico's southern border, currently a highly endemic area for the three arboviruses 7,8 , has been the most affected region due to repeated outbreaks and epidemics that heavily impacted the local health and economy.

The global burden of arboviral diseases and their rapid geographical spread require the formulation of effective and sustainable strategies to reduce the proliferation of viruses and their vectors. Therefore, it is extremely important to understand the nature and interaction of the risk factors behind arboviruses' emergence, reemergence and persistence 1,2,9.

Human practices play a fundamental role in the diseases spread, closely related to domestic and peri-domestic indices of Aedes larval infestation. The wide availability of breeding sites (trashes, residues, disused bottles, disused tires, or any containers that can be filled with rainwater) within domestic environments can be considered an indicator of vector proliferation and disease transmission risk, as they enable the proliferation of the mosquitoes, and therefore the diseases transmission 10.

Vector control, especially at domestic level, is an essential component for arboviral diseases prevention, and the identification of arbovirus-related knowledge and practices contributes for the development of more effective and context-adapted strategies 9 . Yet, more factors must be considered and assessed to prevent arboviral epidemics and endemicity 11 . Since the epidemiological patterns of arboviral diseases are associated with a set of social, ecological, and environmental determinants 9,10,11,12,13,14, the World Health Organization (WHO) strongly recommends the implementation of an integrated vector management for controlling and preventing arboviral diseases 15 . According to the WHO Commission on Social Determinants of Health (CSDH), intermediate determinants are assets or circumstances with great impact on human health by their interrelations with biological, psychosocial and behavioral factors 16,17 .

Arboviral diseases are deeply associated to water, sanitation, and hygiene (WASH) indicators, infrastructure conditions, education level and socioeconomic status $11,12,13,14,18,19,20,21,22,23$. Thus, the aim of this work is to assess the influence of these socio-environmental factors on the knowledge about arboviral diseases and related preventive practices among inhabitants of three rural communities of Southern Mexico. Social and intermediate health determinants such as education, water supply and public hygiene services were investigated to identify possible associations with community knowledge, interpretations and behaviors affecting the vector ecology. 


\section{Methods}

\section{Study setting}

The study was conducted at three coastal rural villages near the southern border of Mexico: Ejido Hidalgo and Ejido Río Florido, of the municipality of Tapachula (state of Chiapas) and Ranchería Guineo Segunda Sección, of the municipality of Villahermosa (state of Tabasco).

These three locations were chosen for sharing socioeconomic, geographical and ecological conditions, presenting Ae. aegypti and Ae. albopictus vectors, as well as being located along chikungunya and Zika south-north expansion since 20158.

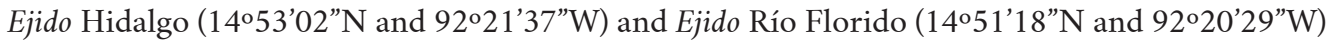
are located at approximately $15 \mathrm{~km}$ southwest of Tapachula city, in the coastal plain of Chiapas at a $56 \mathrm{~m}$ mean altitude. Both Ejidos are separated by a distance of $3.5 \mathrm{~km}$. The predominant climate is tropical monsoon ("Am"), with an average annual temperature of $26.5^{\circ} \mathrm{C}$ and $2,700 \mathrm{~mm}$ of annual precipitations. Ejido Río Florido comprises an area of 20 hectares, 789 inhabitants, 172 inhabited households and 4.59 inhabitants per household. Ejido Hidalgo comprises an area of 24 hectares, 697 inhabitants, 184 inhabited households and 3.79 inhabitants per household. In both villages, people are mainly dedicated to agricultural activities and the cultivation of several commercial crops 24,25 .

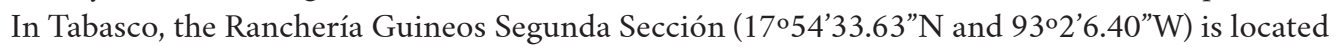
at approximately $13 \mathrm{~km}$ southeast of Villahermosa city, in the alluvial plain of Tabasco, at an $11 \mathrm{~m}$ mean altitude. The predominant climate is "Am", with average annual temperature of $27.3^{\circ} \mathrm{C}$ and about $1,972 \mathrm{~mm}$ of annual precipitations. The village comprises approximately 20 hectares, approximately 1,032 inhabitants, 263 inhabited households and 3.92 inhabitants per household. Most households have electricity and piped water. People are mainly dedicated to agricultural and aquaculture activities 24,26 .

Ejido Hidalgo and Ejido Río Florido on one hand, and Ranchería Guineo Segunda Sección on the other represent two extremes of a DENV, CHIKV and ZIKV prevalence gradient. In 2017, there were over 17,404 confirmed cases of the three diseases in the whole country: 1,240 in Chiapas ( $7 \%$ of the total) and only 98 confirmed cases in Tabasco ( $0.6 \%$ of the total) 7 .

The three localities were setting for a multidisciplinary pilot project focused on arboviruses control and prevention, coordinated by the El Colegio de la Frontera Sur (ECOSUR) 27. The first step was the development of a community engagement plan with local stakeholders of the two Tapachula's villages, to improve community involvement and participation in arboviral diseases control 28 (Supplementary Material 1: http://cadernos.ensp.fiocruz.br/site/public_site/arquivo/suppl-1-e00110519_9667.pdf).

\section{Study design and participants}

The present cross-sectional mixed-method study consisted of two stages:

(1) Pre-intervention: in June 2017, prior to Tapachula's community engagement interventions, one Household Survey (about knowledge and reported practices on arboviruses) and one Risk Observational Assessment (about risk practices for vector proliferation at domestic level) were applied per household.

(2) Post-intervention: between July and August 2018, the Risk Observational Assessment was reapplied in the same households. Furthermore, information regarding predisposing circumstances for vector proliferation at community level (water supply and environmental public health services) was gathered by the application of a Community Background Survey, complemented by a qualitative questionnaire.

The surveys were applied to a set of 149 households, chosen through a simple random sampling process: 82 households in Tapachula municipality (34 from Ejido Río Florido and 48 from Ejido Hidalgo) and 67 households in Villahermosa municipality, totaling 643 residents. Sample size was calculated at $95 \%$ confidence interval $(95 \% \mathrm{CI})$, with $5 \%$ margin of error and $10 \%$ non-response rate.

For the qualitative questionnaire, a purposive sampling process was used to recruit two key informants per community, members of local organizations playing a relevant role in the management of community health problems: the local community health center (Casa de Salud) and the main local government body (Asamblea Ejidal). 
All participants of the study were informed of its purpose and gave their consent. In agreement with article 23 of the Regulation of the General Health Law in the Field of Research of Mexican Ministry of Health, considering the nature of the investigation, only verbal informed consent was applied. The research project proposal was approved by the Ethics Research Committee of ECOSUR, with reference n. CEI-006-2018.

\section{Surveys (Supplementary Material 2; http://cadernos.ensp.fiocruz.br/site/public_site/ arquivo/suppl-2-e00110519_2525.pdf)}

\section{- Household Survey}

A structured, standardized and pre-coded questionnaire was designed to obtain information on the sociodemographic characteristics of the household residents regarding age, schooling level, number of residents per household and average women per household. In addition, residents were asked about their knowledge on arboviruses' origin and transmission, as well as the use of preventive measures. Interviews were conducted using the "breadwinner" as the main respondent.

\section{- Risk Observational Assessment}

In 2017 and 2018, through the application of a structured, standardized and pre-coded household observational checklist, the following information were collected regarding the presence or absence of risk factors for vector proliferation in the households: presence or absence of unprotected water deposits, solid waste accumulation and others risk elements. Observations were made in the households surroundings, or patio (courtyard), a fundamental extension of the house where the kitchen, dining room and rustic bathroom are generally located 29.

\section{- Community Background Survey}

The presence of risk factors for Aedes breeding within the whole community was first assessed through pre-coded questions to household residents. The questions focused on sanitation, hygiene services and infrastructures available in the villages.

Then, a semi-structured questionnaire for the key informants of each community was designed to provide a better insight of information recorded during home visits. In addition, further qualitative data were collected to explore risk perceptions and community's expectations regarding arboviral diseases and their prevention.

\section{Data collection and management}

Fieldwork data collection was performed by trained staff and graduate students from ECOSUR, Andalusian School of Public Health (EASP; Granada, Spain) and Touro University (Vallejo, USA). A representant from the Comisariado Ejidal (representation of the Asamblea Ejidal) of each community accompanied the team during fieldwork to gain a better understanding and acceptance from the rest of the community.

Information were collected using paper questionnaires and quantitative data were entered (twice) into a database, using spreadsheets files. All data files were checked and cleaned separately by field supervisors. Data files of all study stages were first analyzed separately and then merged and analyzed altogether.

Qualitative data from the Community Background Survey were written as field notes, initially by hand, during or directly after interview process. Afterwards, data were transcribed and organized into a data analysis template. 
Knowledge, referred and observed practice score system development (Supplementary

Material 2; http://cadernos.ensp.fiocruz.br/site/public_site/arquivo/

suppl-2-e00110519_2525.pdf)

To evaluate each section of the proposed surveys, indices were designed to score the adequate or inadequate knowledge and practices at stake, as previously done in several studies 30,31,32,33.

Questions regarding knowledge allowed a single correct answer. Each correct answer was rated one point. Questions regarding practices allowed several correct answers so that each interviewee had up to five possible options. Each correct answer was rated 0.5 points. In any case, wrong answers or the lack of answer were rated 0 points. Direct observations, made in interior and exterior spaces of household units, focused on two aspects: presence of unprotected water deposits and detection of organic or inorganic solid waste. One point was assigned when these elements were absent (observed adequate practices) and 0 points if not. A knowledge or practice was considered adequate when the "overall score" was equal or greater than $60 \%$ of the maximum expected score.

\section{Variables of study}

Adequate or inadequate (according to the previous scale) knowledge, referred and observed practices were the dichotomous categorical dependent variables. Independent variables for socio-environmental determinants were: age, schooling level, number of residents per household, average women per household, perceived prevalence of arboviral diseases, presence of sewage system and frequency of water supply and waste collection services.

\section{Statistical analysis}

Univariate and bivariate categorical statistical analysis were performed. As categorical dependent variables had only two possible outcomes, a binary logistic regression model was used to explore associations among independent and dependent variables. Odds ratios (OR) and their respective 95\%CI were derived, and goodness of fit was assessed using the Hosmer-Lemeshow test. Statistical analysis was performed with R 3.2.1 (http://www.r-project.org) and the R Commander package 2.4-4.

\section{Qualitative analysis}

A content analysis approach, following a hybrid process of deductive and inductive analysis, was used to identify common or recurrent patterns among explored themes. Transcriptions were systematically reviewed, organized and coded according to the main themes explored: water, sanitation and hygiene services in the community, arboviral diseases risk and their prevention within the community 34 . Information obtained by qualitative data enabled a triangulation with community background data, collected using the quantitative survey 35 .

\section{Results}

\section{Sociodemographic characteristics}

Table 1 shows the sociodemographic characteristics of the study sample by residence locality. The majority of households had an average of three or more inhabitants (82,5\%). Overall, the most common type of family was "young", represented by a mean age for household members of less than 35 years (55\%). There were no significant differences in schooling level, gender and age distribution among the localities of the two municipalities. 
Table 1

Sociodemographic characteristics (Household Survey and Community Background Survey).

\begin{tabular}{|c|c|c|c|c|c|c|c|}
\hline \multirow[t]{2}{*}{ Characteristics } & \multicolumn{2}{|c|}{ Tapachula $(n=82)$} & \multicolumn{2}{|c|}{ Villahermosa $(n=67)$} & \multicolumn{3}{|c|}{ Global $(n=149)$} \\
\hline & $\mathbf{n}$ & $\%$ & $\mathbf{n}$ & $\%$ & $\mathbf{n}$ & $\%$ & p-value \\
\hline \multicolumn{8}{|l|}{ Residents per household } \\
\hline $1-2$ & 17 & 20.7 & 9 & 13.4 & 26 & 17.4 & 0.503 \\
\hline $3-4$ & 33 & 40.2 & 29 & 43.3 & 62 & 41.6 & \\
\hline$>4$ & 32 & 39.0 & 29 & 43.3 & 61 & 40.9 & \\
\hline \multicolumn{8}{|l|}{ Women per household } \\
\hline $0-1$ & 28 & 34.1 & 20 & 29.9 & 48 & 32.2 & 0.843 \\
\hline $2-3$ & 41 & 50.0 & 35 & 52.2 & 76 & 51.0 & \\
\hline$>3$ & 13 & 15.9 & 12 & 17.9 & 25 & 16.8 & \\
\hline \multicolumn{8}{|l|}{ Average women per household (\%) } \\
\hline$<50$ & 58 & 70.7 & 48 & 71.6 & 106 & 71.1 & 0.902 \\
\hline$\geq 50$ & 24 & 29.3 & 19 & 28.4 & 43 & 28.9 & \\
\hline \multicolumn{8}{|l|}{ Family's age structure } \\
\hline Young (<35 years old) & 40 & 48.8 & 42 & 62.7 & 82 & 55.0 & 0.089 \\
\hline Adult and old ( $\geq 35$ years old) & 42 & 51.2 & 25 & 37.3 & 67 & 45.0 & \\
\hline \multicolumn{8}{|l|}{ Average schooling level ( $\geq 15$ years old) } \\
\hline Uneducated & 11 & 13.4 & 4 & 6.0 & 15 & 10.1 & 0.186 \\
\hline Incomplete elementary & 17 & 20.7 & 12 & 17.9 & 29 & 19.5 & \\
\hline Elementary & 32 & 39.0 & 37 & 55.2 & 69 & 46.3 & \\
\hline Advanced (secondary and higher education) & 22 & 26.8 & 14 & 20.9 & 36 & 24.2 & \\
\hline \multicolumn{8}{|l|}{ Female average education ( $\geq 15$ years old) } \\
\hline Uneducated & 12 & 15.2 & 8 & 11.9 & 20 & 13.7 & 0.919 \\
\hline Incomplete elementary & 16 & 20.3 & 16 & 23.9 & 32 & 21.9 & \\
\hline Elementary & 32 & 40.5 & 27 & 40.3 & 59 & 40.4 & \\
\hline Advanced (secondary and higher education) & 19 & 24.1 & 16 & 23.9 & 35 & 24.0 & \\
\hline \multicolumn{8}{|l|}{ Solid waste collection services } \\
\hline Daily & 0 & 0.0 & 0 & 0.0 & 0 & 0.0 & 0.000 \\
\hline Weekly & 0 & 0.0 & 65 & 97.0 & 65 & 43.3 & \\
\hline Monthly & 82 & 100.0 & 2 & 3.0 & 84 & 56.7 & \\
\hline \multicolumn{8}{|l|}{ Water supply services } \\
\hline Regular & 0 & 0.0 & 0 & 0.0 & 0 & 0.0 & 0.000 \\
\hline Irregular & 0 & 0.0 & 54 & 80.6 & 54 & 36.0 & \\
\hline Absent & 82 & 100.0 & 13 & 19.4 & 95 & 64.0 & \\
\hline \multicolumn{8}{|l|}{ Drainage system } \\
\hline Present & 0 & 0.0 & 0 & 0.0 & 0 & 0.0 & NA \\
\hline Partial & 0 & 0.0 & 0 & 0.0 & 0 & 0.0 & \\
\hline Absent & 82 & 100.0 & 67 & 100.0 & 149 & 100.0 & \\
\hline
\end{tabular}

NA: not available.

\section{Community background contextualization}

As shown in Table 1, significant difficulties in community sanitation were detected, as the frequency of solid waste collection by municipal services was monthly in the two Tapachula's communities and mostly weekly (97\%) in Villahermosa. There was no public sewage system in both communities. A significantly high percentage of irregular water supply was reported in Villahermosa $(80,6 \%)$, whereas completely absent in Tapachula. 
These findings were supported by the key informants during interviews. Furthermore, a high level of annoyance and concern was detected, especially regarding waste management. As reported by a member of the Río Florido Comisariado Ejidal:

P1: "Before, there were no such problems: each family consumed what they had. Now, everything comes in plastic, packaging, and then we do not know what to do with so many things. There is a landfill tax, but it is only for families who can afford it".

Most of the six informants defined the situation as "[institutional] abandonment" and perceived an increased risk of infection for the community. They also mentioned perceiving a greater difficulty in complying with preventive measures.

P1: "More and more people get sick".

P2: "There are more and more mosquitoes"; "They are everywhere"; "We need to stop it".

P3: "This has already become a problem".

Cleaning domestic spaces was stressed as the main preventive measure to be followed. Community participation in this task was described as a fundamental element for prevention effectiveness, although difficult to achieve. As a health staff member expressed:

P2: "The most important thing is to keep the patios [backyards] clean. It is a task of the whole community; we all must do it to make it work. This is why it is very difficult".

Regarding water management, wells (private or communal) were pointed out by all informants as the main solution to precarious supply. In Ejido Hidalgo, it was reported that the lack of a functioning communal well forced families without other resources to store rainwater in domestic containers.

\section{Knowledge and reported vector control practices}

Table 2 describes the scores regarding knowledge and reported preventive practices, as determined by the Household Survey. Nearly all respondents had already heard of the diseases (99.3\%), and knowledge about arboviruses and their transmission was generally good (75.2\%), especially regarding DENV (81.2\%). However, there were only a few cases $(30.7 \%)$ of adequate personal (how to protect themselves from mosquito bites) or domestic (how to avoid mosquitoes breeding in and around households) prevention measures.

\section{Observed vector control practices}

As shown in Table 3, both in 2017 and in 2018, a high proportion of households presented some unprotected water containers in outdoors areas (Tapachula: $73.7 \%$ and $42.1 \%$; Villahermosa: $58.2 \%$ and 54.5\% respectively), as well as waste accumulation (Tapachula: 60.5\% and 65.8\%; Villahermosa: $36.4 \%$ and $30.3 \%$ respectively). In 2017 , Villahermosa's village showed a higher percentage of adequate practices than Tapachula's villages $(56.1 \%$ and $18.4 \%, \mathrm{p}<0.001)$. However, in 2018, Tapachula's villages presented an increase in observed adequate practices, from $18.4 \%$ to $27.6 \%$. Such change is closely associated with a decrease of unprotected water deposits, as the amount of organic and inorganic waste recorded in 2018 was even higher than 2017. In Villahermosa's village, observed adequate practices slightly decreased from $56.1 \%$ to $42.4 \%$, due to the growth of unprotected water deposits and waste in 2018. It is interesting to observe that the percentages of adequate practices in both sites were not statistically different in $2018(\mathrm{p}=0.064)$.

\section{Logistic regression model}

Tables 4 and 5 show the results of logistic models for the association between independent variables for the investigated socio-environmental determinants and reported knowledge and practices. Higher schooling levels were associated with better scores on knowledge and prevention. Household members with incomplete education had 4 times $(\mathrm{OR}=4.13$; 95\%CI: 1.2-13.9) more probability of presenting inadequate knowledge on arboviruses when compared to members with primary or higher education. Likewise, they showed a higher probability of presenting observed inadequate practices, both in 2017 (OR = 38.33; 95\%CI: 7.87-186.53) and 2018 (OR $=15.04$; 95\%CI: 3.89-58.05). 
Table 2

Knowledge and reported practices to prevent arboviral diseases (Household Survey).

\begin{tabular}{|c|c|c|c|c|c|c|c|}
\hline & \multicolumn{2}{|c|}{ Tapachula $(n=82)$} & \multicolumn{2}{|c|}{ Villahermosa $(n=67)$} & \multicolumn{3}{|c|}{ Global $(n=149)$} \\
\hline & $\mathbf{n}$ & $\%$ & $\mathbf{n}$ & $\%$ & $\mathbf{n}$ & $\%$ & p-value \\
\hline \multicolumn{8}{|c|}{ Had heard about DENV, ZIKV, CHIKV } \\
\hline Yes & 81 & 98.8 & 67 & 100.0 & 148 & 99.3 & 0.364 \\
\hline No & 1 & 1.2 & 0 & 0.0 & 1 & 0.7 & \\
\hline \multicolumn{8}{|l|}{ Where? } \\
\hline Television & 20 & 24.7 & 30 & 44.8 & 50 & 33.8 & 0.000 \\
\hline Neighbors & 18 & 22.2 & 14 & 20.9 & 32 & 21.6 & \\
\hline Health center & 17 & 21.0 & 15 & 22.4 & 32 & 21.6 & \\
\hline Disease exposure & 22 & 27.2 & 0 & 0.0 & 22 & 14.9 & \\
\hline Other & 0 & 0.0 & 2 & 3.0 & 2 & 1.4 & \\
\hline \multicolumn{8}{|l|}{ Perceived prevalence } \\
\hline Yes & 62 & 75.6 & 22 & 32.8 & 84 & 56.4 & 0.000 \\
\hline No & 20 & 24.4 & 45 & 67.2 & 65 & 43.6 & \\
\hline \multicolumn{8}{|l|}{ Knowledge } \\
\hline Adequate (global) & 64 & 78.0 & 48 & 71.6 & 112 & 75.2 & 0.368 \\
\hline Inadequate (global) & 18 & 22.0 & 19 & 28.4 & 37 & 24.8 & \\
\hline \multicolumn{8}{|l|}{ Dengue } \\
\hline Adequate & 67 & 81.7 & 54 & 80.6 & 121 & 81.2 & 0.255 \\
\hline Inadequate & 15 & 18.3 & 13 & 19.4 & 28 & 18.8 & \\
\hline \multicolumn{8}{|l|}{ Chikungunya } \\
\hline Adequate & 63 & 76.8 & 49 & 73.1 & 112 & 75.2 & 0.210 \\
\hline Inadequate & 19 & 23.2 & 18 & 26.9 & 37 & 24.8 & \\
\hline \multicolumn{8}{|l|}{ Zika } \\
\hline Adequate & 58 & 70.7 & 43 & 64.2 & 101 & 67.8 & 0.311 \\
\hline Inadequate & 24 & 29.3 & 24 & 35.8 & 48 & 32.2 & \\
\hline \multicolumn{8}{|l|}{ Reported practices } \\
\hline Adequate (global) & 31 & 38.6 & 14 & 20.9 & 45 & 30.7 & 0.019 \\
\hline Inadequate (global) & 51 & 61.4 & 53 & 79.1 & 104 & 69.3 & \\
\hline \multicolumn{8}{|l|}{ Personal protection } \\
\hline Adequate & 13 & 16.2 & 9 & 13.4 & 22 & 15.2 & 0.701 \\
\hline Inadequate & 69 & 84.3 & 58 & 86.6 & 128 & 84.8 & \\
\hline \multicolumn{8}{|l|}{ Home protection } \\
\hline Adequate & 44 & 53.0 & 22 & 32.8 & 66 & 44.4 & 0.013 \\
\hline Inadequate & 38 & 47.0 & 45 & 67.2 & 84 & 55.6 & \\
\hline
\end{tabular}

Moreover, a higher percentage of domestic risk practices was associated with significant deficiencies in sanitation and water supply services. When frequency of solid waste collection was monthly, it was noticed a higher risk of observed inadequate practices than weekly collection $(2017$ : OR $=6.17$; 95\%CI: 2.75-14.52) (2018: OR = 2.09; 95\%CI: 0.99-4.53). Similar results were found regarding the comparison between the lack of a water supply network and its presence, even though irregular (2017: $\mathrm{OR}=6.02$; 95\%CI: 2.69-13.93) (2018: OR = 3.60; 95\%CI: $1.64-8.01)$. 
Table 3

Observed practices (Risk Observational Assessment).

\begin{tabular}{|c|c|c|c|c|c|c|c|c|c|c|}
\hline & \multicolumn{5}{|c|}{2017} & \multicolumn{5}{|c|}{2018} \\
\hline & \multicolumn{2}{|c|}{$\begin{array}{c}\text { Tapachula } \\
(n=76)\end{array}$} & \multicolumn{2}{|c|}{$\begin{array}{l}\text { Villahermosa } \\
\qquad(n=66)\end{array}$} & \multirow[t]{2}{*}{ p-value } & \multicolumn{2}{|c|}{$\begin{array}{c}\text { Tapachula } \\
(n=76)\end{array}$} & \multicolumn{2}{|c|}{$\begin{array}{l}\text { Villahermosa } \\
\qquad(n=66)\end{array}$} & \multirow[t]{2}{*}{ p-value } \\
\hline & $\mathbf{n}$ & $\%$ & $\mathbf{n}$ & $\%$ & & $\mathbf{n}$ & $\%$ & $\mathbf{n}$ & $\%$ & \\
\hline Adequate * & 14 & 18.4 & 37 & 56.1 & 0.000 & 21 & 27.6 & 28 & 42.4 & 0.064 \\
\hline Inadequate ** & 62 & 81.6 & 29 & 43.9 & & 55 & 72.4 & 38 & 57.6 & \\
\hline \multicolumn{11}{|c|}{ Unprotected water deposits } \\
\hline Absent & 20 & 26.3 & 28 & 41.8 & 0.050 & 44 & 57.9 & 30 & 45.5 & 0.138 \\
\hline Present & 56 & 73.7 & 39 & 58.2 & & 32 & 42.1 & 36 & 54.5 & \\
\hline \multicolumn{11}{|c|}{ Solid waste accumulation } \\
\hline Absent & 30 & 39.5 & 42 & 63.6 & 0.375 & 26 & 34.2 & 46 & 69.7 & 0.000 \\
\hline Present & 46 & 60.5 & 24 & 36.4 & & 50 & 65.8 & 20 & 30.3 & \\
\hline
\end{tabular}

* Absence of unprotected water deposits and/or solid waste;

** Presence of water deposits and/or solid waste accumulation.

\section{Table 4}

Respondents' sociodemographic characteristics and reported knowledge and practices in 2017.

\begin{tabular}{|c|c|c|c|c|c|c|c|c|c|c|c|c|c|c|}
\hline \multirow[t]{3}{*}{ Characteristics } & \multicolumn{7}{|c|}{ Knowledge (2017) } & \multicolumn{7}{|c|}{ Reported practices (2017) } \\
\hline & \multicolumn{2}{|c|}{ Adequate } & \multicolumn{2}{|c|}{ Inadequate } & \multirow[t]{2}{*}{ p-value } & \multirow[t]{2}{*}{ OR } & \multirow[t]{2}{*}{$95 \% \mathrm{Cl}$} & \multicolumn{2}{|c|}{ Adequate } & \multicolumn{2}{|c|}{ Inadequate } & \multirow[t]{2}{*}{ p-value } & \multirow[t]{2}{*}{ OR } & \multirow[t]{2}{*}{$95 \% \mathrm{Cl}$} \\
\hline & $\mathbf{n}$ & $\%$ & $\mathbf{n}$ & $\%$ & & & & $\mathbf{n}$ & $\%$ & $\mathbf{n}$ & $\%$ & & & \\
\hline \multicolumn{15}{|l|}{ Residents per household } \\
\hline $1-2$ & 16 & 61.5 & 10 & 38.5 & 0.067 & 1.00 & Reference & 4 & 15.4 & 22 & 84.6 & 0.911 & 1.00 & Reference \\
\hline $3-4$ & 52 & 83.9 & 10 & 16.1 & & 0.30 & $0.10-0.87$ & 11 & 17.7 & 51 & 82.3 & & 0.84 & $0.24-2.93$ \\
\hline$>4$ & 44 & 72.1 & 17 & 27.9 & & 0.62 & $0.23-1.62$ & 9 & 15.0 & 51 & 85.0 & & 1.03 & $0.28-3.70$ \\
\hline \multicolumn{15}{|l|}{ Women per household } \\
\hline $0-1$ & 31 & 64.6 & 17 & 35.4 & 0.003 & 1.00 & Reference & 9 & 18.8 & 39 & 81.2 & 0.463 & 1.00 & Reference \\
\hline $2-3$ & 66 & 86.8 & 10 & 13.2 & & 0.27 & $0.11-0.67$ & 13 & 17.3 & 62 & 82.7 & & 1.10 & $0.43-2.81$ \\
\hline$>3$ & 15 & 60.0 & 10 & 40.0 & & 1.21 & $0.44-3.29$ & 2 & 8.0 & 23 & 92.0 & & 2.65 & $0.52-13.36$ \\
\hline \multicolumn{15}{|c|}{ Average women per household (\%) } \\
\hline$<50$ & 32 & 74.4 & 11 & 25.6 & 0.892 & 1.00 & Reference & 9 & 20.9 & 34 & 79.1 & 0.319 & 1.00 & Reference \\
\hline$\geq 50$ & 80 & 75.5 & 26 & 24.5 & & 0.94 & $0.42-2.13$ & 15 & 14.3 & 90 & 85.7 & & 1.58 & $0.63-3.96$ \\
\hline \multicolumn{15}{|c|}{ Family's age structure (average age) } \\
\hline Young (<35 years old) & 63 & 76.8 & 19 & 23.2 & 0.603 & 1.00 & Reference & 14 & 17.3 & 67 & 82.7 & 0.698 & 1.00 & Reference \\
\hline Adult ( $\geq 35$ years old) & 49 & 73.1 & 18 & 26.9 & & 1.22 & $0.57-2.56$ & 10 & 14.9 & 57 & 85.1 & & 1.19 & $0.49-2.88$ \\
\hline \multicolumn{15}{|c|}{ Average education ( $\geq 15$ years old) } \\
\hline Uneducated * & 29 & 65.9 & 15 & 34.1 & 0.057 & 4.13 & $1.23-13.9$ & 13 & 36.1 & 23 & 63.9 & 0.072 & 0.81 & $0.90-3.49$ \\
\hline Elementary & 51 & 73.9 & 18 & 26.1 & & 2.82 & $0.87-9.09$ & 15 & 21.7 & 54 & 78.3 & & 2.03 & $0.83-4.95$ \\
\hline Advanced ** & 32 & 88.9 & 4 & 11.1 & & 1.00 & Reference & 18 & 40.9 & 26 & 59.1 & & 1.00 & Reference \\
\hline \multicolumn{15}{|c|}{$\begin{array}{l}\text { Female average education ( } \geq 15 \\
\text { years old) }\end{array}$} \\
\hline Uneducated * & 28 & 69.2 & 16 & 30.8 & 0.492 & 1.77 & $0.64-4.91$ & 20 & 38.5 & 32 & 61.5 & 0.400 & 1.07 & $0.42-2.72$ \\
\hline Elementary & 45 & 76.3 & 14 & 23.7 & & 1.24 & $0.44-3.46$ & 16 & 27.1 & 43 & 72.9 & & 0.64 & $0.25-1.60$ \\
\hline Advanced ** & 28 & 80.0 & 7 & 20.0 & & 1.00 & Reference & 10 & 28.6 & 25 & 71.4 & & 1.00 & Reference \\
\hline
\end{tabular}

(continues) 
Table 4 (continued)

\begin{tabular}{|c|c|c|c|c|c|c|c|c|c|c|c|c|c|c|}
\hline \multirow[t]{3}{*}{ Characteristics } & \multicolumn{7}{|c|}{ Knowledge (2017) } & \multicolumn{7}{|c|}{ Reported practices (2017) } \\
\hline & \multicolumn{2}{|c|}{ Adequate } & \multicolumn{2}{|c|}{ Inadequate } & \multirow[t]{2}{*}{ p-value } & \multirow[t]{2}{*}{ OR } & \multirow[t]{2}{*}{$95 \% \mathrm{Cl}$} & \multicolumn{2}{|c|}{ Adequate } & \multicolumn{2}{|c|}{ Inadequate } & \multirow[t]{2}{*}{$p$-value } & \multirow[t]{2}{*}{ OR } & \multirow[t]{2}{*}{$95 \% \mathrm{Cl}$} \\
\hline & $\mathbf{n}$ & $\%$ & $\mathbf{n}$ & $\%$ & & & & $\mathbf{n}$ & $\%$ & $\mathbf{n}$ & $\%$ & & & \\
\hline \multicolumn{15}{|c|}{ Solid waste collection services } \\
\hline Monthly & 64 & 78.0 & 18 & 22.0 & 0.367 & 1.40 & $0.66-2.96$ & 33 & 38.8 & 52 & 61.2 & 0.013 & 0.04 & $0.17-0.87$ \\
\hline Weekly & 48 & 71.6 & 19 & 28.4 & & 1.00 & Reference & 13 & 20.0 & 52 & 80.0 & & 1.00 & Reference \\
\hline \multicolumn{15}{|c|}{ Water supply services } \\
\hline Absent & 72 & 75.8 & 23 & 24.2 & 0.815 & 0.91 & $0.40-2.14$ & 34 & 35.4 & 62 & 64.6 & 0.092 & 0.52 & $0.22-1.18$ \\
\hline Irregular & 40 & 74.1 & 14 & 25.9 & & 1.00 & Reference & 12 & 22.2 & 42 & 77.8 & & 1.00 & Reference \\
\hline \multicolumn{15}{|c|}{ Perceived prevalence } \\
\hline Yes & 66 & 78.6 & 18 & 21.4 & 0.274 & 1.00 & Reference & 13 & 15.7 & 70 & 84.3 & 0.836 & 1.00 & Reference \\
\hline No & 46 & 70.8 & 19 & 29.2 & & 1.51 & $0.71-3.20$ & 11 & 16.9 & 54 & 83.1 & & 0.91 & $0.37-2.19$ \\
\hline
\end{tabular}

95\% Cl: 95\% confidence interval; OR: odds ratio.

* Without schooling or incomplete;

** Secondary and higher.

Table 5

Respondents' sociodemographic characteristics and observed practices in 2017 and 2018.

\begin{tabular}{|c|c|c|c|c|c|c|c|c|c|c|c|c|c|c|}
\hline \multirow[t]{3}{*}{ Characteristics } & \multicolumn{7}{|c|}{ Observed practices (2017) } & \multicolumn{7}{|c|}{ Observed practices (2018) } \\
\hline & \multicolumn{2}{|c|}{ Adequate } & \multicolumn{2}{|c|}{ Inadequate } & \multirow[t]{2}{*}{ p-value } & \multirow[t]{2}{*}{ OR } & \multirow[t]{2}{*}{$95 \% \mathrm{Cl}$} & \multicolumn{2}{|c|}{ Adequate } & \multicolumn{2}{|c|}{ Inadequate } & \multirow[t]{2}{*}{ p-value } & \multirow[t]{2}{*}{ OR } & \multirow[t]{2}{*}{$95 \% \mathrm{Cl}$} \\
\hline & $\mathbf{n}$ & $\%$ & $\mathbf{n}$ & $\%$ & & & & $\mathbf{n}$ & $\%$ & $\mathbf{n}$ & $\%$ & & & \\
\hline \multicolumn{15}{|l|}{ Residents per household } \\
\hline $1-2$ & 3 & 11.5 & 23 & 88.5 & 0.009 & 1.00 & Reference & 6 & 24.0 & 19 & 76.0 & 0.418 & 1.00 & Reference \\
\hline $3-4$ & 22 & 36.7 & 38 & 63.3 & & 0.22 & $0.06-0.83$ & 20 & 35.1 & 37 & 64.9 & & 0.58 & $0.20-1.69$ \\
\hline$>4$ & 26 & 46.4 & 30 & 53.6 & & 0.15 & $0.04-0.55$ & 23 & 39.0 & 36 & 61.0 & & 0.49 & $0.17-1.42$ \\
\hline \multicolumn{15}{|l|}{ Women per household } \\
\hline $0-1$ & 1 & 23.9 & 35 & 76.1 & 0.073 & 1.00 & Reference & 12 & 26.1 & 34 & 73.9 & 0.320 & 1.00 & Reference \\
\hline $2-3$ & 28 & 38.9 & 44 & 61.1 & & 0.49 & $0.21-1.12$ & 27 & 38.6 & 43 & 61.4 & & 0.56 & $0.25-1.27$ \\
\hline$>3$ & 12 & 50.0 & 12 & 50.0 & & 0.31 & $0.11-0.89$ & 10 & 40.0 & 15 & 60.0 & & 0.53 & $0.18-1.49$ \\
\hline \multicolumn{15}{|c|}{ Average women per household (\%) } \\
\hline$<50$ & 11 & 26.8 & 30 & 73.2 & 0.561 & 1.00 & Reference & 10 & 25.0 & 30 & 75.0 & 0.125 & 1.00 & Reference \\
\hline$\geq 50$ & 40 & 39.6 & 61 & 60.4 & & 0.55 & $0.25-1.24$ & 39 & 38.6 & 62 & 61.4 & & 0.53 & $0.20-1.27$ \\
\hline \multicolumn{15}{|c|}{ Family's age structure (average age) } \\
\hline Young (<35 years old) & 36 & 46.8 & 41 & 53.2 & 0.003 & 1.00 & Reference & 34 & 44.2 & 43 & 55.8 & 0.010 & 1.00 & Reference \\
\hline Adult ( $\geq 35$ years old) & 15 & 23.1 & 50 & 76.9 & & 2.90 & $1.33-6.55$ & 15 & 23.4 & 49 & 76.6 & & 2.56 & $1.17-5.80$ \\
\hline \multicolumn{15}{|c|}{ Average education ( $\geq 15$ years old) } \\
\hline Uneducated * & 3 & 4.8 & 39 & 95.2 & 0.000 & 38.3 & $7.87-186.53$ & 3 & 7.3 & 38 & 92.7 & 0.000 & 15.00 & $3.89-58.05$ \\
\hline Elementary & 26 & 40.0 & 39 & 60.0 & & 2.87 & $1.22-6.76$ & 27 & 41.5 & 38 & 58.5 & & 1.67 & 0.73-3.82 \\
\hline Advanced ** & 23 & 65.7 & 12 & 34.3 & & 1.00 & Reference & 19 & 54.3 & 16 & 45.7 & & 1.00 & Reference \\
\hline \multicolumn{15}{|c|}{$\begin{array}{l}\text { Female average education ( } \geq 15 \\
\text { years old) }\end{array}$} \\
\hline Uneducated * & 5 & 10.2 & 44 & 89.8 & 0.000 & 51.00 & $13.56-192.06$ & 0 & 0.0 & 49 & 100.0 & & & \\
\hline Elementary & 17 & 30.4 & 39 & 69.6 & & 13.30 & $4.40-40.24$ & 22 & 40.0 & 33 & 60.0 & & & \\
\hline Advanced ** & 29 & 85.3 & 5 & 14.7 & & 1.00 & Reference & 26 & 76.5 & 8 & 23.5 & & 1.00 & Reference \\
\hline
\end{tabular}

(continues) 
Table 5 (continued)

\begin{tabular}{|c|c|c|c|c|c|c|c|c|c|c|c|c|c|c|}
\hline \multirow[t]{3}{*}{ Characteristics } & \multicolumn{7}{|c|}{ Observed practices (2017) } & \multicolumn{7}{|c|}{ Observed practices (2018) } \\
\hline & \multicolumn{2}{|c|}{ Adequate I } & \multicolumn{2}{|c|}{ Inadequate } & \multirow[t]{2}{*}{ p-value } & \multirow[t]{2}{*}{ OR } & \multirow[t]{2}{*}{$95 \% \mathrm{Cl}$} & \multicolumn{2}{|c|}{ Adequate } & \multicolumn{2}{|c|}{ Inadequate } & \multirow[t]{2}{*}{ p-value } & \multirow[t]{2}{*}{ OR } & \multirow[t]{2}{*}{$95 \% \mathrm{Cl}$} \\
\hline & $\mathbf{n}$ & $\%$ & $\mathbf{n}$ & $\%$ & & & & $\mathbf{n}$ & $\%$ & $\mathbf{n}$ & $\%$ & & & \\
\hline \multicolumn{15}{|c|}{ Solid waste collection services } \\
\hline Monthly & 14 & 17.9 & 64 & 82.1 & 0.000 & 6.17 & $2.75-14.52$ & 21 & 26.9 & 57 & 73.1 & 0.035 & 2.09 & $0.99-4.53$ \\
\hline Weekly & 37 & 57.8 & 27 & 42.2 & & 1.00 & Reference & 28 & 43.8 & 36 & 56.2 & & 1.00 & Reference \\
\hline \multicolumn{15}{|c|}{ Water supply services } \\
\hline Absent & 18 & 20.5 & 70 & 79.5 & 0.000 & 6.02 & $2.69-13.93$ & 21 & 23.6 & 68 & 76.4 & 0.000 & 3.60 & $1.64-8.01$ \\
\hline Irregular & 33 & 61.1 & 21 & 38.9 & & 1.00 & Reference & 28 & 52.8 & 25 & 47.2 & & 1.00 & Reference \\
\hline \multicolumn{15}{|c|}{ Perceived prevalence } \\
\hline Yes & 24 & 30.0 & 56 & 70.0 & 0.095 & 1.00 & Reference & 30 & 38.5 & 48 & 61.5 & 0.303 & 1.00 & Reference \\
\hline No & 27 & 43.5 & 35 & 56.5 & & 0.55 & $0.26-1.17$ & 19 & 30.2 & 44 & 69.8 & & 1.44 & $0.67-3.13$ \\
\hline
\end{tabular}

95\% Cl: 95\% confidence interval; OR: odds ratio.

* Without schooling or incomplete;

** Secondary and higher.

\section{Discussion}

\section{Key findings and other studies comparison}

Although the study population presented high scores for knowledge on DENV, CHIKV and ZIKV transmission, the overall score for self-reported and observed practices was generally low. Similar findings have been previously reported in other localities with recent history of arboviral diseases $20,33,36,37,38,39$. These outcomes suggest that knowledge does not necessarily lead to adequate prevention practices, especially in contexts where the complex interactions among environmental and social determinants increase human vulnerability to vector borne diseases 12,13,14,40.

Our findings show a high percentage of domestic risk practices associated with great deficiencies in water supply and sanitation services. Among households where these intermediate social determinants were more deteriorated, there was a higher risk of predisposing behaviors for arboviruses proliferation.

Water and sanitation infrastructure are significant determinants for vector breeding risk, and should be considered for the development of strategies for intervention and evaluation of vector control 11,17. High rates of household water storage and increased density of Aedes mosquitoes have been described as related to low socioeconomic conditions, unplanned urbanization and poor water supply 19,20,21,22,23,41,42. In Mexico's context, flaws in water and waste services coverage have been identified as key factors for arboviruses endemicity and the persistence of recurrent outbreaks 43 .

According to the presented results, the community engagement plan performed in Tapachula partially modified the percentage of domestic risk practices. Observations made in 2018, after the community engagement plan, revealed a decrease in the accumulation of unprotected household water deposits. Nevertheless, waste accumulation within domestic environments persisted and even increased. Seemingly, the potential community engagement plan impact could have been affected by Tapachula's villages contextual circumstances, such as: monthly solid waste collection, absence of water supply services and non-functioning communal well. In Villahermosa's village, where no community engagement plan was operated, sanitation and water supply services are more frequently provided than in Tapachula, possibly the reason why Villahermosa's residents showed better practices than Tapachula's.

The responsibility of the whole community in arboviral diseases prevention, as well as the needed action on a problem perceived as very urgent, were recurrent themes on key informants' interviews. Although community involvement was highlighted as a key element for vector control, it was also perceived as something difficult to achieve. The process of community participation in vector control 
activities is described as difficult because it requires time and resources 11 , possibly being more fragile in under-privileged contexts with inadequate infrastructures 21.

Maintaining good hygiene conditions in households and their surroundings was identified as the main practice to prevent the diseases spread, a concept previously described in other studies conducted in Mexico regarding community's perspectives on dengue and its prevention 44,45,46.

Furthermore, a major difficulty in translating preventive measures into everyday actions and behaviors was reported, in accordance with similar findings 21,47,48.

When asked about irregularities in water supply and sanitation services, participants' reported feeling highly exposed to the diseases, as previously mentioned in the context of Tapachula city 45 . Such dimensions are perceived as barriers to encourage community participation in vector control strategies.

Community engagement strategies are complex processes that require a broad understanding of the context, both before and after its implementation. Although evaluating arbovirus-related knowledge, behaviors and perceptions enable the identification of community's needs and expectations, more indicators should be considered and addressed to improve vector control strategies 11 .

Decreasing Aedes vector population from domestic environments is an essential step to reduce the risk of arboviral diseases occurrence 8,9,13 and empowered processes of community-based interventions are potential strategies to effectively and sustainably achieving this goal 9,10,49,50. Nonetheless, characteristics of environments and public infrastructures, as health determinants fundamental parts, could significantly persuade people's ability to perform preventive behaviors and measures 16 . If the impact of these contextual circumstances on people's behaviors and perceptions is not properly considered and addressed, achieving a real change in prevention practices could be a difficult process $11,12,13,14,22,40$.

\section{Limitations of the study}

The reduced sample size and data sparsity regarding surveys quantitative information may possibly have reduced the statistical power for some associations inferential analysis. Surveys regarding knowledge and practices, due to their extension and quantitative approach, do not encourage a deeper understanding of these dimensions and their interaction. Likewise, the categorization of survey data using a scoring system may not be the most appropriate method to approach such a complex issue, difficult to measure and quantify. Information obtained from the "breadwinner" may not always be completely representative of the whole household unit. Inter-observer variability may have affected the information obtained by the Risk Observational Assessment.

\section{Conclusions and implications}

The endemicity of arboviral infection in Southern Mexico results of complex interactions among vector, socio-environmental determinants and human behaviors. To achieve strategies of an effective, sustainable and ethical vector control, a more integrated and contextualized approach is required. Community empowerment is more than just providing information: it is about facilitating and encouraging stakeholders to take control of their actions, through strategies adapted to local conditions, culture and needs. Furthermore, intermediate social determinants of water supply and sanitation services need to be addressed to reduce community vulnerability to arboviral diseases. 


\section{Contributors}

All authors contributed to the drafting and correction of the manuscript. R. Solís-Hernández contributed to the statistical analysis.

\section{Additional informations}

ORCID: Roberta Causa (0000-0003-1567-5441); Héctor Ochoa-Díaz-López (0000-0002-84214983); Ariane Dor (0000-0002-3483-9547); Fernando Rodríguez-León (0000-0003-1360-0577); Roberto Solís-Hernández (0000-0002-5662-7430); Ana Laura Pacheco-Soriano (0000-0002-59222260).

\section{Acknowledgments}

We are thankful to Miguel Ángel Luque Fernández, whose contributions have been essential for the development of this work. We would also like to thank the staff of the EASP XXXIII Public Health Master and the colleagues from ECOSUR Health Department, especially Alberto Fernandez, Rosario García Miranda and César Irecta. We are very grateful to the residents of Ejido Hidalgo, Ejido Rio Florido and Ranchería Guineo Segunda Sección, for all the support, contributions and time they have offered.

\section{References}

1. Kraemer MUG, Sinka ME, Duda KA, Mylne AQN, Shearer FM, Barker CM, et al. The global distribution of the arbovirus vectors Aedes aegypti and Ae. albopictus. Elife 2015; 4:e08347.

2. Leta S, Beyene TJ, De Clercq EM, Amenu K, Kraemer MU, Revie CW. Global risk mapping for major diseases transmitted by Aedes aegypti and Aedes albopictus. Int J Infect Dis 2018; 67:25-35.

3. Rey JR, Lounibos LP. Ecología de Aedes aegypti y Aedes albopictus en América y transmisión de enfermedades. Biomédica 2015; 35:177-85.

4. Patterson J, Sammon M, Garg M. Dengue, Zika and chikungunya: emerging arboviruses in the new world. West J Emerg Med 2016; 17:671-9.

5. Díaz-González EE, Kautz TF, Dorantes-Delgado A, Malo-García IR, Laguna-Aguilar M, Langsjoen RM, et al. First report of Aedes aegypti transmission of chikungunya virus in the Americas. Am J Trop Med Hyg 2015; 93:1325-9.

6. Guerbois M, Fernandez-Salas I, Azar SR, Danis-Lozano R, Alpuche-Aranda CM, Leal G, et al. Outbreak of Zika virus infection, Chiapas State, Mexico, 2015, and first confirmed transmission by Aedes. J Infect Dis 2016; 214:1349-6.

7. Dirección General de Epidemiología, Secretaria de Salud. Información epidemiológica. https://www.gob.mx/salud/acciones-y-pro gramas/informacion-epidemiologica (accessed on 22/May/2019).

8. Ochoa H, Rodríguez León F, Estrada J, Pérez de León A. Dengue, chikungunya y Zika: panorama actual en México y la frontera sur. In: Ochoa H, editor. La Frontera Sur de México, ¿una salud en crisis? Ciudad de México: Academia Nacional de Medicina de México; 2018. p. 91-114.

9. Pan American Health Organization; World Health Organization. Strategy for arboviral disease prevention and control. http://iris.paho.org/xmlui/bitstream/han dle/123456789/31430/CD55-16-e.pdf (accessed on 22/May/2019).

10. Focks DA, Alexander N, Villegas E. Multicountry study of Aedes aegypti pupal productivity survey methodology: findings and recommendations. https://www.who.int/tdr/ publications/documents/aedes_aegypti.pdf (accessed on 22/May/2019).

11. Degroote S, Zinszer K, Ridde V. Interventions for vector-borne diseases focused on housing and hygiene in urban areas: a scoping review. Infect Dis Poverty 2018; 7:96.

12. Bardosh KL, Ryan SJ, Ebi K, Welburn S, Singer B. Addressing vulnerability, building resilience: community-based adaptation to vectorborne diseases in the context of global change. Infect Dis Poverty 2017; 6:166.

13. Gardner LM, Bóta A, Gangavarapu K, Kraemer MUG, Grubaugh ND. Inferring the risk factors behind the geographical spread and transmission of Zika in the Americas. PLoS Negl Trop Dis 2018; 12:e006194. 
14. Roiz D, Wilson AL, Scott TW, Fonseca DM, Jourdain F, Müller $\mathrm{P}$, et al. Integrated $A e$ des management for the control of Aedesborne diseases. PLoS Negl Trop Dis 2018; 12:e0006845.

15. Pan American Health Organization. Vectors: integrated management and public health entomology. https://www.paho.org/hq/index. php?option $=$ com_topics \&view $=$ article $\& i d=$ $65 \&$ Itemid $=40780 \&$ lang $=$ en $($ accessed on 22/ May/2019).

16. Solar O, Irwin A. A conceptual framework for action on the social determinants of health. Geneva: World Health Organization; 2010. (Discussion Paper Series on Social Determinants of Health, 2).

17. Morano JP, Holt DA. The social determinants of health contextualized for the Zika virus. Int J Infect Dis 2017; 65:142-3.

18. World Health Organization. Water, sanitation and hygiene. Strategy 2018-2025. https://apps.who.int/iris/bitstream/han dle/10665/274273/WHO-CED - PHE WSH-18.03-eng.pdf?ua $=1$ (accessed on 22/ May/2019).

19. Ruiz-Díaz MS, Mora-García GJ, SalguedoMadrid GI, Alario A, Gómez-Camargo DE. Analysis of health indicators in two rural communities on the Colombian Caribbean coast: poor water supply and education level are associated with water-related diseases. Am J Trop Med Hyg 2017; 97:1378-92.

20. Arunachalam N, Tana S, Espino F, Kittayapong P, Abeyewickreme W, Wai KT, et al. Eco-biosocial determinants of dengue vector breeding: a multicountry study in urban and periurban Asia. Bull World Health Organ 2010; 88:173-84.

21. Caprara A, Lima JWO, Marinho ACP, Calvasina PG, Landim LP, Sommerfeld J. Irregular water supply, household usage and dengue: a bio-social study in the Brazilian northeast. Cad Saúde Pública 2009; 25 Suppl 1:S25-36.

22. Quintero J, Carrasquilla G, Suárez R, González C, Olano VA. An ecosystemic approach to evaluating ecological, socioeconomic and group dynamics affecting the prevalence of Aedes aegypti in two Colombian towns. Cad Saúde Pública 2009; 25 Suppl 1:S93-103.

23. Spiegel JM, Bonet M, Ibarra AM, Pagliccia N, Ouellette V, Yassi A. social and environmental determinants of Aedes aegypti infestation in Central Havana: results of a case-control study in an integrated dengue surveillance programme in Cuba. Trop Med Int Health 2007; 12:503-10.

24. Secretaría de Desarrollo Social. Catálogo de localidades. http://www.microrregiones. gob.mx/catloc/Default.aspx (accessed on 22/ May/2019).

25. Instituto Nacional de Estadística, Geografía e Informática. Tapachula, Chiapas (07089). https://www.inegi.org.mx/ (accessed on 22/ May/2019).
26. Instituto Nacional de Estadística, Geografía e Informática. Centro, Tabasco (27004). https://www.inegi.org.mx/ (accessed on 22/ May/2019).

27. Dor A, Bond Compeán JG, Casas M, Fernández Salas I, Fernández Marina C, Jamboos Toledo JC. Mosquitos transmisores de arbovirosis emergentes de relevancia en salud pública. In: Ochoa H, editor. La Frontera Sur de México, ¿una salud en crisis? Ciudad de México: Academia Nacional de Medicina de México; 2018. p. 115-29.

28. Dor A, Irecta C, Pacheco AL. Zancudos, enfermedades y participación comunitaria. Ecofronteras 2018; 22:22-5.

29. Tapia-Conyer R, Méndez-Galván J, BurciagaZúñiga P. Community participation in the prevention and control of dengue: the Patio Limpio strategy in Mexico. Paediatr Int Child Health 2012; 32 Suppl 1:10-3.

30. Dégallier N, Vilarinhos PT, de Carvalho MS, Knox MB, Caetano J. People's knowledge and practice about dengue, its vectors, and control means in Brasilia (DF), Brazil: its relevance with entomological factors. J Am Mosq Control Assoc 2000; 16:114-23.

31. Castro M, Sánchez L, Pérez D, Sebrango C, Shkedy Z, Van der Stuyft P. The relationship between economic status, knowledge on dengue, risk perceptions and practices. PLoS One 2013; 8:e81875.

32. Haenchen SD, Hayden MH, Dickinson KL, Walker K, Jacobs EE, Brown HE, et al. Mosquito avoidance practices and knowledge of arboviral diseases in cities with differing recent history of disease. Am J Trop Med Hyg 2016; 95:945-53.

33. Shuaib F, Todd D, Campbell-Stennett D, Ehiri J, Jolly PE. Knowledge, attitudes and practices regarding dengue infection in Westmoreland, Jamaica. West Indian Med J 2010; 59:139-46.

34. Ulin PR. Robinson ET, Tolley EE. Investigación aplicada en salud pública: métodos cualitativos. Washington DC: Pan American Health Organization; 2006.

35. O'Cathain A, Murphy E, Nicholl J. Three techniques for integrating data in mixed methods studies. BMJ 2010; 341:c4587.

36. Koenrdaat CJM, Tuiten, W, Sithiprasasna R, Kijchalao U, Jones JW, Scott TW. Dengue knowledge and practices and their impact on Aedes aegypti populations in Kanphaeng Phet, Thailand. Am J Trop Med Hyg 2006; 74:692700 .

37. Morales AG, Sala AMI. Level of knowledge, attitudes and practices on the prevention of mosquito Aedes aegypti in communities of Diez de Octubre municipality, La Habana. Rev Cubana Hig Epidemiol 2011; 49:247-59. 
38. Espinoza Gómez F, Hernández Suárez CM, Coll Cárdenas R. Factores que modifican los índices larvarios de Aedes aegypti en Colima, México. Rev Panam Salud Pública 2001; 10:6-12.

39. Menchaca-Armenta I, Ocampo-Torres M, Hernández-Gómez A, Zamora-Cerritos K. Risk perception and level of knowledge of diseases transmitted by Aedes aegypti. Rev Inst Med Trop São Paulo 2018; 60:e10.

40. Cartaxo MF, Silva SMD, Silva JG, Beltrão EI, Brayner FA, de Lima Filho JL, et al. Social determinants of health associated with topical repellent use in pregnancy: a cross-sectional study during a Zika outbreak in Brazil. Trans R Soc Trop Med Hyg 2018; 113:65-73.

41. Lozano RD, Rodrigues MH, Avilia M. Gender related family and Aedes aegypti larval breeding risk in Southern Mexico. Salud Pública Méx 2002; 44:237-42.

42. Fuentes-Vallejo M, Higuera-Mendieta DR, García-Betancourt T, Alcalá-Espinosa LA, García-Sánchez D, Munévar-Cagigas DA, et al. Territorial analysis of Aedes aegypti distribution in two Colombian cities: a chorematic and ecosystem approach. Cad Saúde Pública 2015; 31:517-30.

43. Cifuentes E. Factores ambientales que determinan la aparición de brotes y la persistencia del dengue en Morelos. Salud Pública Méx 2007; 49:114-6.

44. Jones CH, Benítez-Valladares D, GuillermoMay G, Dzul-Manzanilla F, Che-Mendoza A, Barrera-Pérez M, et al. Use and acceptance of long lasting insecticidal net screens for dengue prevention in Acapulco, Guerrero, Mexico. BMC Public Health 2014; 14:846-56.
45. Caballero Hoyos R, López TT, Villarreal FC, Lucatero AP, González MA, Coutiño BL. Concepciones culturales sobre el dengue en contextos urbanos de México. Rev Saúde Pública 2006; 40:126-33.

46. Torres Lopez TM, Caballero Hoyos R, Barraza Salas JH, Romero Paredes JJ. Cultural conceptions about dengue in Nayarit, Mexico. Dengue Bull 2006; 30:223-33.

47. Weldon CT, Riley-Powell AR, Aguerre IM, Celis Nacimento RA, Morrison AC, Oberhelman RA, et al. "Zika is everywhere": a qualitative exploration of knowledge, attitudes and practices towards Zika virus among women of reproductive age in Iquitos, Peru. PLoS Negl Trop Dis 2018; 12:e006708.

48. Souza KR, Santos MLR, Guimarães ICS, Ribeiro GDS, Silva LK. Saberes e práticas sobre controle do Aedes aegypti por diferentes sujeitos sociais na cidade de Salvador, Bahia, Brasil. Cad Saúde Pública 2018; 34:e00078017.

49. Castro M, Sánchez L, Pérez D, Carbonell N, Lefèvre P, Vanlerberghe V, et al. A community empowerment strategy embedded in a routine dengue vector control programme: a cluster randomised controlled trial. Trans R Soc Trop Med Hyg 2012; 106:315-21.

50. Andersson N, Nava-Aguilera E, Arosteguí J, Morales-Perez A, Suazo-Laguna H, LegorretaSoberanis J, et al. Evidence based community mobilization for dengue prevention in Nicaragua and Mexico (Camino Verde, the Green Way): cluster randomized controlled trial. BMJ 2015; 351:h3267. 


\section{Resumen}

La proliferación de los arbovirus y sus vectores está influenciada por la interacción compleja entre vectores, medio ambiente y comportamientos humanos. El objetivo de este estudio es analizar la influencia de los determinantes socioambientales sobre el conocimiento y las prácticas relacionadas con la transmisión de los arbovirus entre los residentes de tres comunidades en la frontera sur de México. Entre junio de 2017 y agosto de 2018, se estudiaron 149 domicilios en las comunidades de Tapachula (Chiapas) y Villahermosa (Tabasco). El estudio tuvo como base la aplicación de un proyecto de prevención comunitaria, utilizando diferentes encuestas y abordajes metodológicos. Se usaron odds ratios para estimar las asociaciones entre determinantes socioambientales y el conocimiento $y$ prácticas para el control de la transmisión de los arbovirus, con el uso de regresión logística y técnicas cualitativas. Cerca de un $75 \%$ de los domicilios mostraban conocimiento adecuado sobre el origen $y$ transmisión de los arbovirus, pero solamente un 30\% habian adoptado prácticas apropiadas. Las prácticas de riesgo en los domicilios estaban asociadas a deficiencias significativas en los servicios de saneamiento y abastecimiento de agua. Además, se detectó la percepción de un mayor riesgo $y$ de dificultad en adoptar medidas preventivas. El conocimiento adecuado no necesariamente conduce a prácticas preventivas adecuadas. Los determinantes sociales intermedios influencian la persistencia de comportamientos que actúan como factores de riesgo para la proliferación de los arbovirus. La gestión de vectores eficaz y sostenible es necesaria para lidiar con esos aspectos interrelacionados.

Arbovirus; Control de Mosquitos; Medio Ambiente y Salud Pública; Saneamiento

\section{Resumo}

A proliferação dos arbovírus e seus vetores é influenciada pela interação complexa entre vetores, meio ambiente e comportamentos humanos. O objetivo do estudo é de analisar a influência dos determinantes socioambientais sobre o conhecimento e as práticas relacionados à transmissão dos arbovírus entre os residentes de três comunidades na fronteira sul do México. Entre junho de 2017 e agosto de 2018, foram estudados 149 domicílios nas comunidades de Tapachula (Chiapas) e Villahermosa (Tabasco). O estudo teve como base a aplicação de um projeto de prevenção comunitária, utilizando diferentes inquéritos e abordagens metodológicas. Foram usadas odds ratios para estimar as associações entre determinantes socioambientais e conhecimento e práticas para controle da transmissão dos arbovírus, com o uso de regressão logística e técnicas qualitativas. Cerca de 75\% dos domicílios mostravam conhecimento adequado sobre a origem e transmissão dos arbovírus, mas apenas 30\% haviam adotado práticas apropriadas. As práticas de risco nos domicílios estavam associadas a deficiências significativas nos serviços de saneamento e abastecimento de água. Além disso, foi detectada a percepção de maior risco e de dificuldade em adotar medidas preventivas. $\mathrm{O} c \mathrm{c}$ nhecimento adequado não necessariamente leva a práticas preventivas adequadas. Os determinantes sociais intermediários influenciam a persistência de comportamentos que agem como fatores de risco para a proliferação dos arbovírus. A gestão de vetores eficaz e sustentável é necessária para lidar com esses aspectos interrelacionados.

Arbovirus; Controle de Mosquitos; Meio Ambiente e Saúde Pública; Saneamento
Submitted on 18/Jun/2019

Final version resubmitted on 10/Sep/2019

Approved on 19/Nov/2019 